UCRL- 101436

PREPRINT

\title{
HIGH PRESSURE STUDIES OF PLANETARY MAT'ER
}

\author{
MARVIN ROSS \\ University of California \\ Lawrence Livermore National Laboratory \\ Livermore, CA 94550 USA
}

This paper was prepared for submittal to the

International Conference on High Pressure Science and Technology

July 17-21, 1989

Paderborn, West Germany

June 1989

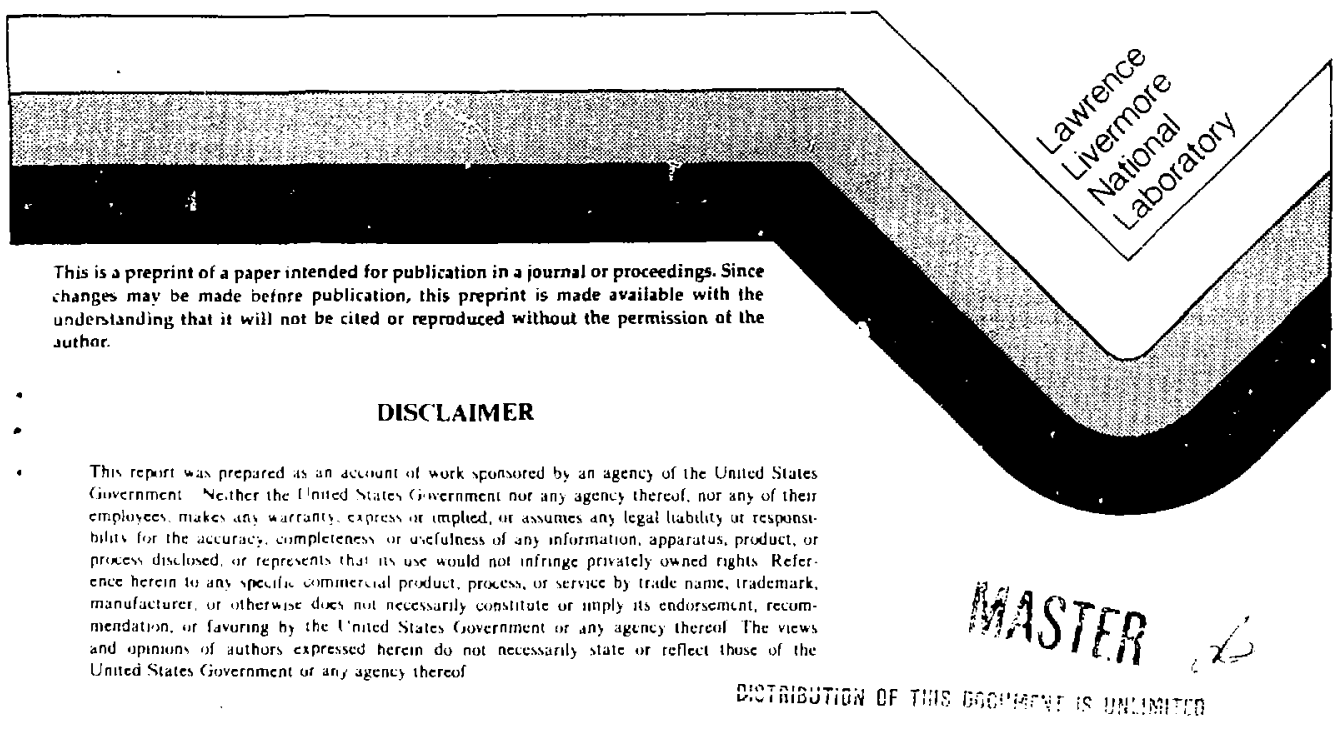


UCRL-- 101436

DE89 017738

\title{
HIGH PRESSURE STUDIES OF PLANETARY MATTER
}

\author{
MARVIN ROSS \\ Lawrence Livermore National Laboratory, University of California \\ Livermore, CA 94550 USA
}

Those materials which are of greatest interest to the physics of the deep planetary interiors are $\mathrm{Fe}, \mathrm{H}_{2}$, He and the Ices. These are sufficiently diverse and intensively studied to offer an overview of present day high pressure research.

KEY WORDS: Planets, Iron, Hydrogen, Helium, Ices.

\section{INTRODUCTION}

For the purpose of discussion the planets may be categorized into two groups: The terrestrial planets, Earth, Mars, Mercury and Venus which are made up of the rocky and solid-like material (Fe, metal oxides, silicates etc.) and the Jovian planets, Jupiter, Saturn, Uranus and Neptune, consisting of an inner rock core estimated to be about fifteen to twenty earth masses surrounded by a large volume of dense fluid. Jupiter and Saturn are made mainly of a solar mixture of $\mathrm{H}_{2}$ and $\mathrm{He}\left(7\right.$ parts $\mathrm{H}_{2}$ to $\mathrm{l}$ part He by number). Uranus and Neptine which are smaller and are at lower temperatures have retained considerable amounts of Ice in addition to their primordial $\mathrm{H}_{2}$ and $\mathrm{He}$. "Ice," is a term used to denote a planetary mixture of $\mathrm{H}_{2} \mathrm{O}, \mathrm{NH}_{3}$ and $\mathrm{CH}_{4}$. Pluto is believed to be mainly made of Ice. A useful survey on the subject of planetary interiors is the book by Hubbard (1984).

\section{PHASE DIAGRAM OF IRON}

The melting pressure and temperature for iron are of great importance for constraining the temperature at the center of the earth and has been tise stimulus for several recent and important advances in static and shockwave methods. Noteworthy are the sound velocity shock melting measurements of Brown and McQueen (1986), the attempts by Williams ef al., (1987), and of Boehler (1986), to determine the melting curve by laser heating an iron simple in a diamond-anvil cell and the shock temperature measurements of Bass (1987).

Boehler reported diamond arvil measurements of the $\varepsilon-\gamma$ (hcp-fcc) and melting curves to $40 \mathrm{GPa}$. Figure 1 shows the phase diagram of iror, combining the results of Boehler with the sound velocity measurements of Brown and McQueen. Boehler predicted that the $\gamma$ phase ends in a tripie point near $75 \mathrm{GPa}$, and he speculated that the shock anomaly at $200 \mathrm{GPa}$ must correspond to a new high pressure solid phase.

Theoretical calculations (Ross et al., 1989), using the Young and Grover (1983) model for iron, predict that this anomaly may be caused by a new high temperature bcc phase, referred to here as $\alpha^{i}$. This model predicts that the cp-bcc $\left(\varepsilon-\alpha^{\prime}\right)$ phase boundary shown in Figure 1 has 


\section{MARVIN ROSS}

a positive $d T / d P$ slope. The titeoretical shock Hugoniot crosses the theoretical $\mathrm{cp}-\mathrm{bcc}$ phase boundary at $130 \mathrm{GPa}$ and $2400 \mathrm{~K}$. Although this differs from the $200 \mathrm{GPa}$ experimental shock anomaly, all considered the agreement is good. If one assumes the calculated $c p-b c c$ phase boundery slope is correct and shifts the boundary to intersect the shock anomaly at $200 \mathrm{GPa}$, then the first appearance of the new bcc phase is predicted to occur on the melting curve at $150 \mathrm{GPa}$ and $4000 \mathrm{~K}$.

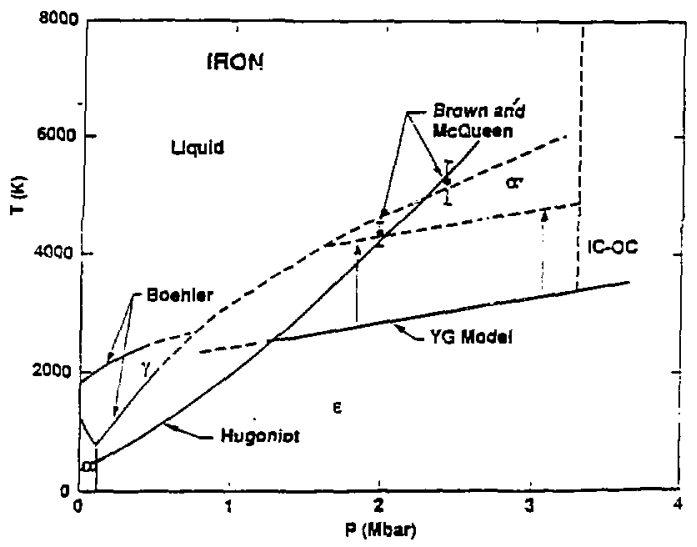

Figure 1. Phase diagram of iron up to Earth core conditions. The experimental phase boundaries of Boehler are shown to $400 \mathrm{kbar}$ and are extrapolated to a $\varepsilon$ - $y$-liquid triple point. The melting curve is then smoothly drawn through the Brown-McQueen shock anomaly at $2.43 \mathrm{Mbar}$. The theoretical cp-bcc: $\left(\varepsilon-\alpha^{\prime}\right)$ phase boundary from the YG model is shown. and the arrows indicate a uniform shift necessary to have this boundary intersect the 2.0 Mbar shock anomaly. IC-OC dashed line indicates pressure at inner- outer-core boundary.

\section{THE JOVIAN PLANETS}

The low mean densities of Jupiter and Saturn lead us to believe they consist mostly of hydrogen and helium near solar composition. Using equations of state based on shock-wave experiments for $\mathrm{H}_{2}$ (Ross et al., 1983) and He (Ross and Young, 1986), with an inner rock core, the internal structure of Jupiter can be modeled to explain all of its observed properties, while assuming solar composition of constituent elements (Grossman et al., 1980). Saturn remains anomalous. Calculations show this body has a cooling time that is too long (it is emitting more energy than can be accounted for by contraction) and which may be the result of energy released by the gravitational differentiation of phase separated hydrogen and helium (Stevenson, 1975). 


\section{HIGH PRESSURE STUDIES OF PLANETARY MATTER}

The simplest models of Uranus and Neptune postulate three layers in which the innermost layer consists of a rock-core of $\mathrm{FeO}$ and the outer is mainly $\mathrm{H}_{2}$ and $\mathrm{He}$. The middle layer is presumed rich in the intermediate mass elements present in accordance with their cosmochemical ratios, $\mathrm{O}: \mathrm{C}: \mathrm{N}(7: 4: 1)$, and in the form of their hydrides $\mathrm{H}_{2} \mathrm{O}$, $\mathrm{CH}_{4}$ and $\mathrm{NH}_{3}$.

The middle layer of Uranus, and Neptune, are of particular interest because a part of their P-T range is attainable by current experimental techniques. The pressures and temperatures calculated for this layer range from about $20 \mathrm{GPa}$ and $200 \mathrm{~K}$ at the outer layer boundary, to about $600 \mathrm{GPa}$ and $6000 \mathrm{~K}$ at the ice/rock core boundary (Hubbard, 1984). Existing shock-wave data up to nearly 1 Mbar indicates that above this pressure the molecules believed to constitute the ice layer of Uranus and Neptune are highly ionized, and almost completely dissociated. This means that the middle layer is divided into an ionized molecular outer-part and an inner-part at higher temperature and higher pressure which is dissociated and becomes progressively metallic at higher densities.

\section{ACKNOWLEDGEMENTS}

Work performed under auspices of U.S. DOE by Lawrence Livermore National Laboratory under contract number W-7405-ENG-48.

\section{REFERENCES}

Bass, J. D. in High-Pressure Research in Mineral Physics (eds. Marighnani, M. H. and Syono, Y.) 393-402 (Terra Scientific, Tokyo, 1987).

Boehler, R. Geophys. Res. Lett. 13, 1153-1156 (1986).

Brown, J. J. and McQueen, R. G. J. Geophys. Res. 21, 7485-7494 (1986).

Grossman, A. S., Pollack, J. B., Reynolds, R. T., Summers, A. L., and

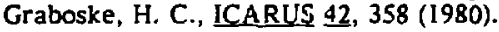

Hubbard, W. B., Planetary Interiors, Van Nostrand Reinhold Co. (1984).

Ross, M., Ree, F. H., and Young, D. A., J.Chem. Phys. 79. 1487 (1983).

Ross, M. and Young, D. A., Phys. Lett. A.18, 463 (1986).

Ross, M., Young, D. A., and Grover, R., submitted for publication (1989).

Stevenson, D. J., Phys. Rey. 12B, 3999-4007 (1975).

Williams, Q., Jeanloz, R., Bass, J. D., Svendsen, B., and Ahrens, T. J. Science 236, 181-182 (1987).

Young, D. A. and Grover, R. in Shock Waves in Condensed Matter - 1983 (eds Asay, J. R., Graham, R. A., and Straub, G. K.) 65-67 (North Holland, Amsterdam 1984). 